\title{
Line Shape Diagnostics for Solid Density Plasmas Produced by Ultra Intense Subpicosecond Laser
}

\author{
Z.Jiang,* J.C.Kieffer, M.Chaker \\ INRS-energie, 1650 montee Ste-Julie, Varennes, Canada \\ G.Korn, S.Coe, G.Mourou \\ CUOS, Univ. of Michigan, Ann Arbor, USA \\ O.Peyrusse, D.Gilles \\ CEA Limeil, Villeneuve, St.Georges, France
}

The recent development of a new generation of lasers has opened up new horizons in laser matter interaction. ${ }^{1}$ The short duration of the pulse effectively eliminates the plasma hydrodynamics during the heating phase. Furthermore if there is negligible energy in the prepulse, the density gradient scale length will be much shorter than the wavelength and the laser-matter coupling will take place at near solid density plasmas. The 1D, hot, uniform solid density plasma offers a unique way to address experimentally in the laboratory some problems: i) of astrophysical interest, namely the opacity of dense matter and the physics of hot strongly correlated plasmas ${ }^{2}$ ii)of interest for atomic physics, for instance the physics of quasi-molecular states ${ }^{3}$ and iii)of application interests, as the fast electron ignition ${ }^{4}$ in ICF and the generation of ultrafast $x$-ray source. 5,6

Recently we have conducted several experiments to study the behaviors of solid density plasmas with an ultraclean (contrast ratio of about $10^{-10: 1)} 300 \mathrm{fs}$ laser pulse at intensity up to a few times $10^{18} \mathrm{~W} / \mathrm{cm}^{2}$. Time and space integrated line profiles of $\mathrm{He}$-like and $\mathrm{Li}$-like emissions are used to infer the average plasma density at which various emissions take place by using Stark broadening calculations ${ }^{7}$ (with the ions and the electrons treated in, respectively, the quasi static and impact approximation). One aspect of the line shape calculations is the use of analytic fits of the plasma microfield distribution function, deduced from a large number of Monte Carlo simulation. These analytical fits depend on the ionic correlation parameters and on the electron screening and thus the microfield will be very sensitive to the ratio of electron to ion temperature $\left(T_{e} / T_{i}\right)$.

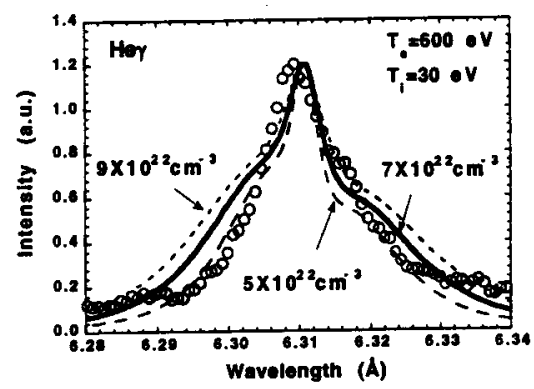

Fig.l Al He rline shape (dot) and fits with different densities. $I_{L}=10^{18} \mathrm{~W} / \mathrm{cm}^{2}$.

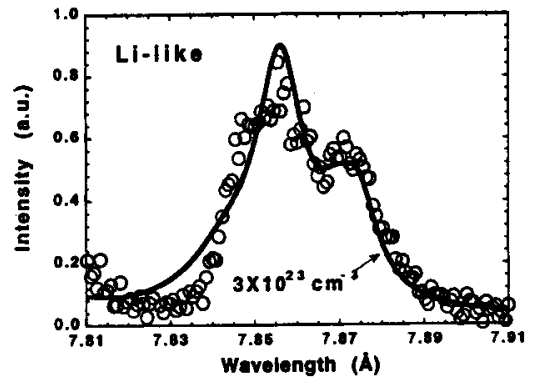

Fig.2 Al Li-like line shape (dot) and fit (solid line). $I_{L}=10^{18} \mathrm{~W} / \mathrm{cm}^{2}$.

Fig. 1 shows a Her line profile obtained at laser intensity of $10^{18} \mathrm{~W} / \mathrm{cm}^{2}$. The best fit (solid line) gives $\mathrm{T}_{\mathrm{i}}=50 \mathrm{eV}$ and $\mathrm{n}_{\mathrm{e}}=5 \times 10^{22} \mathrm{~cm}^{-3}$. We checked that this 
couple of parameters allows also a good fit of the $\mathrm{He} \beta$ line profile.The time average electron temperature is estimated as $600 \mathrm{eV}$ by using $\mathrm{Ly} \alpha / \mathrm{He} \beta$ line ratios calculated with time dependent collisional radiative code TRANSPEC. 8

The density at which the Li-like emission takes place has been inferred from the $1 \mathrm{~s} 212 \mathrm{l}^{\prime}-1 \mathrm{~s}^{2} 2 \mathrm{l}$ line spectra. ${ }^{7}$ At the electron density larger than $10^{23} \mathrm{~cm}^{-3}$, the broadening is important and double excited level populations have reached the local thermodynamic equilibrium (LTE). Fig. 2 shows a Li-like spectrum taken at the laser intensity of $10^{18 \mathrm{~W}} / \mathrm{cm}^{2}$. The best calculation fit is also shown $\left(3 \times 10^{23} \mathrm{~cm}^{-3}\right)$.

Fig. 3 presents the variation of the density inferred from He-like and Lilike line shapes for Al.The density for He-like emission is constant with the laser intensity, and the inferred ion temperature is always smaller than the electron temperature. The density for Li-like emission remains constant in the intensity range $10^{16} \mathrm{~W} / \mathrm{cm}^{2}$ to $3 \times 10^{17} \mathrm{~W} / \mathrm{cm}^{2}$, increases up to a maximum $\left(4 \times 10^{23} \mathrm{~cm}^{-3}\right)$ at intensity around $10^{18} \mathrm{~W} / \mathrm{cm}^{2}$ and decreases dramatically after the peak.

The results suggest the radiative pressure of the powerful laser pulse

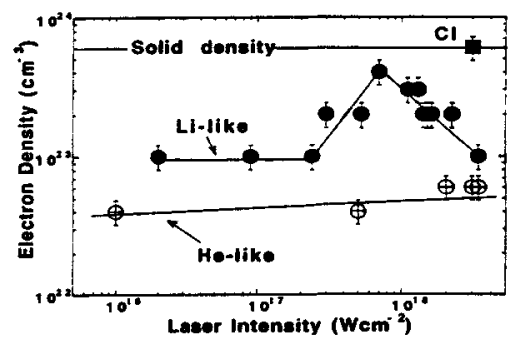

Fig.3 The variation of the density inferred from $\mathrm{He}$-like (open dots) and Li-like (full dots) shapes for Al. The square is the density from $\mathrm{Cl}$ Li-Like line shapes. plays a very important role. The pressure could balance the thermal pressure and maintain a very steep gradient during the coupling thus forcing the emission to be produced at near solid density. But at the higher intensity the Al plasma is overdriven and the emission is produced during the plasma decompression on a time length longer than the laser pulse. Results obtained with a high $\mathrm{Z}$ target $(\mathrm{Cl}$ Li-like emission ) indicate that when the target atomic number is matched to the electron temperature, the emission is produced at solid density (square in Fig.3) at $3 \times 10^{18} \mathrm{~W} / \mathrm{cm}^{2}$. This behavior indicates that there is an optimum range set by the target material and laser intensity to produced $\mathrm{KeV}$ emission at solid density.

This work is supported by NSERC, FCAR and Ministere de l'education du Quebec.

\section{Reference}

* On leave from Shanghai Institute of Optics and Fine Mechanics, China

1. M.Perry and G.Mourou, Sciences 264, 917(1994)

2. F.Rogers and C.Iglesias, Science 263, 50(1994)

3. E.Leboucher-Dalimier, A.Poquerusse and P.Angelo, Phys.Rev.E47, 1467(1993)

4. M.Tabak, J.Hammer, M.Glinsky, W.Kruer, S.Wilks, J.Woodworth, E.Michael and M.Perry, Phys.Plasmas 1, 1626(1994)

5. J.C.Kieffer, M.Chaker, J.Matte, H.Pepin, C.Y.Cote, Y.Beaudoin, T.Johnston, C.Y.Chien, S.Coe, G.Mourou and O.Peyrusse, Phys.Fluids B5, 2676(1993)

6. M.M.Murnane, H.C.Kapteyn and R.W.Falcone, Phys.Rev.Lett.62, 155(1989)

7. O.Peyrusse, J.C.Kieffer, C.Y.Cote and M.Chaker, J.Phys.B:At.Mot.Opt.Phys.26, L51 1(1993)

8. O.Peyrusse, Phys.Fluids B4, 2007(1992) 